\title{
Termination of ongoing spike-wave discharges investigated by cortico-thalamic network analyses
}

\author{
Annika Lüttjohann ${ }^{\mathrm{a}, *}$, Jan-Mathijs Schoffelen ${ }^{\mathrm{b}, \mathrm{c}}$, Gilles van Luijtelaar ${ }^{\mathrm{a}}$ \\ ${ }^{a}$ Radboud University Nijmegen, Donders Institute for Brain, Cognition and Behaviour, Donders Centre for Cognition, Nijmegen, The Netherlands \\ ${ }^{\mathrm{b}}$ Radboud University Nijmegen, Donders Institute for Brain, Cognition and Behaviour, Donders Centre for Cognitive Neuroimaging, Nijmegen, The Netherlands \\ c Max Planck Institute for Psycholinguistics, Nijmegen, The Netherlands
}

\section{A R T I C L E I N F O}

\section{Article history:}

Received 13 February 2014

Revised 19 May 2014

Accepted 9 June 2014

Available online 18 June 2014

\begin{abstract}
A B S T R A C T
Purpose: While decades of research were devoted to study generation mechanisms of spontaneous spike and wave discharges (SWD), little attention has been paid to network mechanisms associated with the spontaneous termination of SWD. In the current study coupling-dynamics at the onset and termination of SWD were studied in an extended part of the cortico-thalamo-cortical system of freely moving, genetic absence epileptic WAG/Rij rats.

Methods: Local-field potential recordings of 16 male WAG/Rij rats, equipped with multiple electrodes targeting layer 4 to 6 of the somatosensory-cortex (ctx4, ctx5, ctx6), rostral and caudal reticular thalamic nucleus (rRTN \& cRTN), ventral postero medial (VPM), anterior- (ATN) and posterior (Po) thalamic nucleus, were obtained. Six seconds lasting pre-SWD- > SWD, SWD- > post SWD and control periods were analyzed with timefrequency methods, and between-region interactions were quantified with frequency-resolved Granger Causality (GC) analysis.

Results: Most channel pairs showed increases in GC lasting from onset to offset of the SWD. While for most thalamo-thalamic pairs a dominant coupling direction was found during the complete SWD, most corticothalamic pairs only showed a dominant directional drive (always from cortex to thalamus) during the first $500 \mathrm{~ms}$ of SWD. Channel pair ctx4-rRTN showed a longer lasting dominant cortical drive, which stopped $1.5 \mathrm{sec}$ prior to SWD offset. This early decrease in directional coupling was followed by an increase in directional coupling from cRTN to rRTN $1 \mathrm{sec}$ prior to SWD offset. For channel pairs ctx5-Po and ctx6-Po the heightened cortex- > thalamus coupling remained until $1.5 \mathrm{sec}$ following SWD offset, while the thalamus- > cortex coupling for these pairs stopped at SWD offset.

Conclusion: The high directional coupling from somatosensory cortex to the thalamus at SWD onset is in good agreement with the idea of a cortical epileptic focus that initiates and entrains other brain structures into seizure activity. The decrease of cortex to rRTN coupling as well as the increased coupling from cRTN to rRTN preceding SWD termination demonstrates that SWD termination is a gradual process that involves both cortico-thalamic as well as intrathalamic processes. The rostral RTN seems to be an important resonator for SWD and relevant for maintenance, while the cRTN might inhibit this oscillation. The somatosensory cortex seems to attempt to reinitiate SWD following its offset via its strong coupling to the posterior thalamus.
\end{abstract}

(c) 2014 Elsevier Inc. All rights reserved.

\section{Introduction}

Absence epilepsy (AE) is a neurological disorder, mostly found in young children, which is characterized by frequent, spontaneously starting and spontaneously stopping, lapses of consciousness. The major electrophysiological characteristic of AE are the spontaneously starting and stopping, rhythmic, generalized, bilateral synchronous spike and wave discharges (SWD), which are known to be generated

\footnotetext{
* Corresponding author at: Institute of Physiology I, Westfälische Wilhelms University Münster, Robert-Koch Str. 27a, 48149 Münster, Germany.

E-mail address: annika.luettjohann@ukmuenster.de (A. Lüttjohann).

Available online on ScienceDirect (www.sciencedirect.com).
}

within the cortico-thalamo-cortical system (Depaulis and van Luijtelaar, 2006).

While decennia of research have been devoted to explore the generation mechanisms of SWD, little attention has been devoted to why and how ongoing SWD spontaneously terminate, although knowledge of such intrinsic stopping mechanisms might result in the development of new therapeutic strategies.

Sohal et al. (2003) as well as Proulx et al. (2006) proposed that the reticular thalamic nucleus (RTN) might be involved in the control of SWD duration. They showed that selective pharmacological manipulations, which affect the intra RTN communication, significantly shortened SWD. Sitnikova et al. (2008) studied the interaction between the frontal cortex and VPM with a non-frequency resolved linear estimation 
of Granger Causality (GC) during transition periods surrounding the onset and offset of SWD. They reported a gradual decrease in corticothalamic coupling, which started prior to the end of SWD and a strong thalamo-cortical coupling which remained present throughout the SWD. It was proposed that the reduced influence of the cortex on the thalamus might be related to the termination of SWD, whereas the thalamus (VPM) is not much involved in SWD termination.

For SWD generation, however, there are more/other structures known to be involved. Here, rather than the frontal cortex, the deep somatosensory cortex seems to be crucial, which has been proposed to contain an excitable epileptic focus (SWD instigator zone) (Lüttjohann et al., 2011; Meeren et al., 2002; Polack et al., 2007) in genetic absence epileptic WAG/Rij and GAERS rats, two well validated animal models of absence epilepsy (Coenen and Van Luijtelaar, 2003; Depaulis and van Luijtelaar, 2006; Marescaux et al., 1992).

Regarding the thalamus, next to the VPM also the posterior thalamus (Po) (Kostopoulos, 2001; Lüttjohann and van Luijtelaar, 2012; Polack et al., 2007) was proposed to be crucial while the RTN classically assumed to be relevant for sleep spindles and SWD (Meeren et al., 2005; Steriade, 2003) can no longer be seen as a homogeneous structure but appears to have opposite functions for its caudal and rostral part (Aker et al., 2006; Meeren et al., 2009).

All these structures need to interact in a particular fashion so that an SWD can arise: despite decades of debate on whether the cortex or the thalamus is the initiator of SWD, it is today more and more assumed, that a focal zone (in deep somatosensory cortex in rats) stimulates various parts of the thalamus (VPM, Po, and RTN), eliciting both excitation and inhibition during the SWD. The latter occurs via the RTN's feedforward synaptic inhibition of thalamo-cortical cells (Huguenard and McCormick, 2007; Meeren et al., 2002; Pinault and O'Brien, 2005; Steriade, 1998). Like SWD generation, also the termination of SWD might rely on a particular interaction of these structures.

In the current study we investigate how all these structures, known to be important for SWD generation, interact during the spontaneous termination of SWD. Local field potentials of SWD - > post-SWD transition periods were obtained from this extended part of the corticothalamo-cortical system of absence epileptic WAG/Rij rats (including the deep layers of the somatosensory cortex, rostral and caudal RTN, Po, VPM and the anterior thalamic nucleus (ATN)). Signals were analyzed by a frequency resolved Granger Causality analysis, a directed connectivity analysis, which infers causal relationships between signals (Dhamala et al., 2008a,b) and reveals whether there is a dominant structure (cortex or a thalamic nucleus) that 'drives' the termination of SWD.

To get a more complete picture also pre-SWD - > SWD transition periods were analyzed. Since the same dataset as in previous studies on SWD generation was used regarding the onset of SWD (Lüttjohann and van Luijtelaar, 2012; Lüttjohann et al., 2013), this also suited to validate previous network results on SWD generation using another directed connectivity analysis and to extend it to changes in network interactions associated with the termination of SWD.

\section{Methods}

\section{Subjects}

Sixteen male WAG/Rij rats, 6 to 9 months of age were used as experimental subjects. They were born and raised at the department of Biological Psychology, Donders Centre for Cognition, Radboud University Nijmegen, The Netherlands. Prior to surgery rats were housed in pairs (High Makrolon ${ }^{\circledR}$ cages with Enviro Dri® bedding material and cage enrichment) with free access to food and water and were kept at a 12-12 h light-dark cycle (light off at 8.30 AM). After surgery rats were housed individually. The experiment was approved by the Ethical Committee on Animal Experimentation of Radboud University
Nijmegen (RU-DEC). Efforts were made to keep the discomfort for the animals as minimal as possible.

\section{Surgery}

Implantation of the LFP recording electrodes was done in a stereotactic frame under isoflurane anesthesia. At the start of surgery, rats received a subcutaneous injection of the analgesic Rimadyl ${ }^{\circledR}$ and an intramuscular injection of atropine to prevent excessive salivary production. Body temperature was controlled and conserved via a heating pad. The local anesthetic Lidocaine was used on the incision points. Holes were drilled into the skull on top of the right hemisphere for the insertion of recording electrodes at the following positions: Somatosensory cortex: $\mathrm{A} / \mathrm{P}=0$, $\mathrm{M} / \mathrm{L}=-4.6$ depth $=-2.8$ (layer 4$),-3.1$ (layer 5), $-3.6($ layer 6 ); anterior thalamus: $\mathrm{A} / \mathrm{P}=-1.4 \mathrm{M} / \mathrm{L}=-1$, depth $=-6.2$; rostral RTN: A/P $=-1.4, \mathrm{M} / \mathrm{L}=-1.9$, depth $=-6.6$; posterior thalamic nucleus: $\mathrm{A} / \mathrm{P}=-3.6, \mathrm{M} / \mathrm{L}=-2$, depth $=-5.4$; VPM: $\mathrm{A} / \mathrm{P}=-4.16$, $\mathrm{M} / \mathrm{L}=-2.8$, depth $=-6$ and caudal RTN: $\mathrm{A} / \mathrm{P}=-3.1, \mathrm{M} / \mathrm{L}=-3.5$, depth $=-6.6$. All coordinates were determined relative to Bregma according to the rat-brain atlas of Paxinos and Watson (1998). Electrode wires, assembled in a self-constructed electrode system (Lüttjohann and van Luijtelaar, 2012; Lüttjohann et al., 2013; van Luijtelaar et al., 2011) were simultaneously inserted into the brain. Ground and reference electrodes were positioned epidurally on top of the cerebellum. The electrode assembly was fixed to the skull via dental cement. Postoperative analgesic Rimadyl ${ }^{\circledR}$ ( 24 and 48 hours after surgery) was administered, and rats were allowed to recover for two weeks.

\section{Recording of local field potentials}

Two weeks after surgery rats were placed individually in a $20 \times 35 \times 25$ inch Plexiglas registration box and connected to the recording leads for multi-channel LFP recordings. These were attached to a swivel-contact, which allowed recording in freely moving animals. The LFP signals were amplified with a physiological amplifier (TD 90087, Radboud University Nijmegen, Electronic Research Group), filtered by a band pass filter with cut-off points at 1 (high pass) and 100 (low pass) and a $50 \mathrm{~Hz}$ Notch filter, and digitized with a constant sample rate of $2048 \mathrm{~Hz}$ on a WINDAQ recording system (DATAQ-Instruments). The movements of the rat were registered by means of a Passive Infrared Registration system (PIR, RK2000DPC LuNAR PR Ceiling Mount, Rokonet). Each rat was recorded for a period of 4 hours during the dark phase of the light-dark cycle.

\section{Signal analysis}

A subset of 10 pre-ictal - > ictal transition periods, and 10 ictal - > post ictal transition periods of 6 seconds duration were selected for each rat for signal analysis. Transition periods were taken from SWD with a minimal duration of 6 seconds and separated by at least 8 seconds to avoid overlap between different analysis windows.

As in earlier studies (Lüttjohann and van Luijtelaar, 2012; Lüttjohann et al., 2013) the onset of the SWD in the pre-ictal - > ictal transition period was represented by the occurrence of the first epileptic cortico-thalamic spike (FCTS). This is defined as a first sharp spike of at least twice the amplitude of background LFPs, visible in all cortical and thalamic recordings, which is followed by rhythmic SWD activity (see for example Fig. 1 or (Lüttjohann and van Luijtelaar, 2012; Lüttjohann et al., 2013)). Likewise the last sharp spike of at least twice the background amplitude of LFPs, visible in all cortical and thalamic recordings, which is preceded by rhythmic SWD activity, was defined as moment of SWD offset (LCTS) (Fig. 1).

Selected epochs of non-epileptic activity were used as control data. For each rat ten epochs of 500 ms duration were randomly selected during passive wakefulness distant (by at least five minutes) to SWD. 

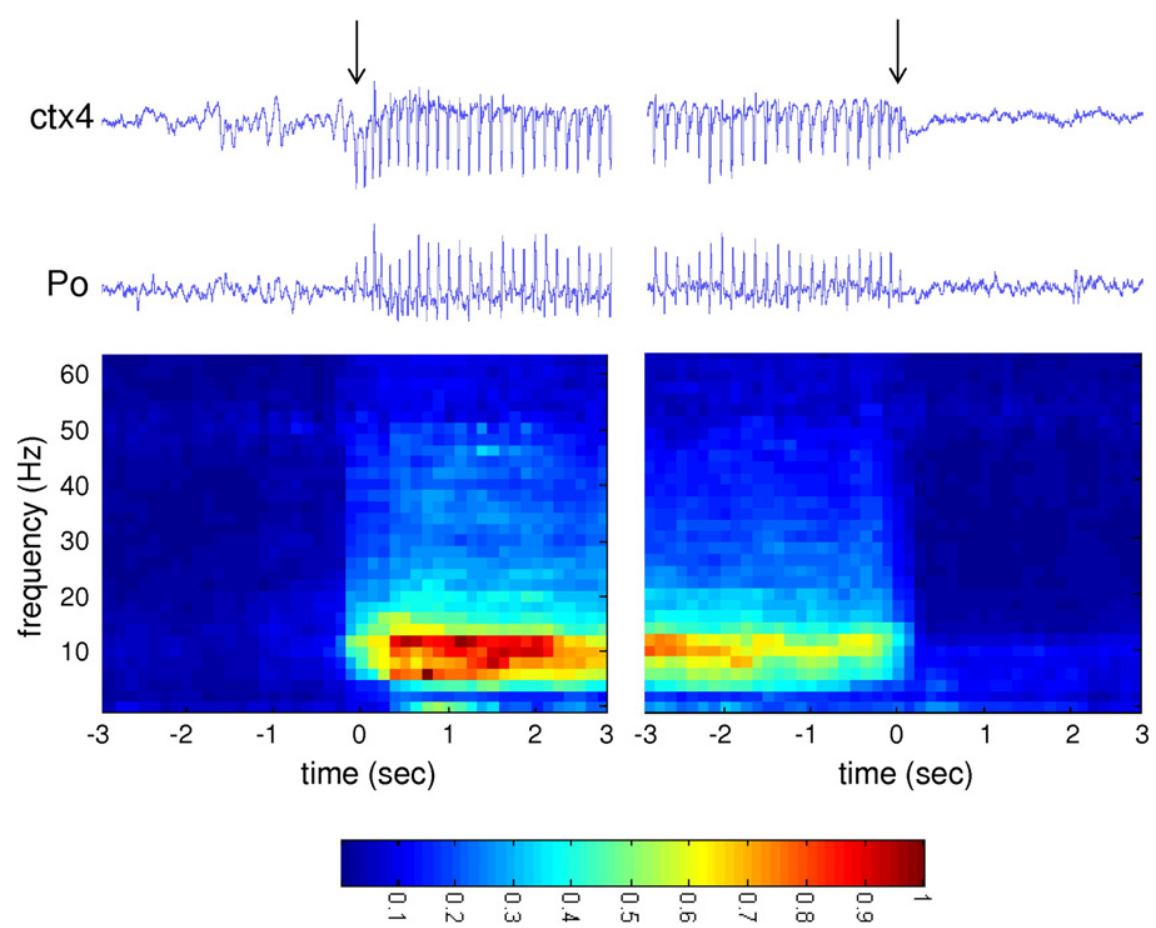

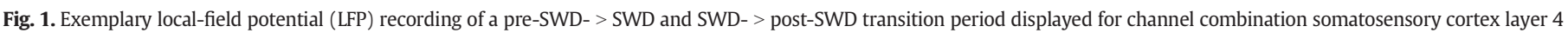

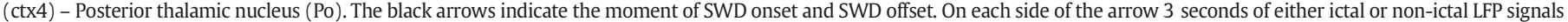

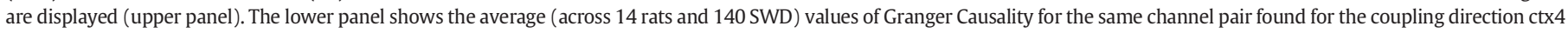
- > Po.

Passive wakefulness is characterized by low amplitude, desynchronized, high frequency LFP signals recorded in a motionless rat. A major proportion of SWD tend to emerge during this state of vigilance (Drinkenburg et al., 1991).

Ten epochs (per rat) of 500 ms duration, taken in the middle of an SWD, functioned as a 'stable SWD' control.

It can be noted that the same control periods as well as the same preictal- > ictal transition periods served as a dataset in earlier connectivity studies (Lüttjohann and van Luijtelaar, 2012; Lüttjohann et al., 2013), investigating network changes occurring with the onset of SWD. This enabled a direct comparison between results with respect to network changes at SWD onset.

Since, for SWD, changes in connectivity are known to occur within timeframes as short as $500 \mathrm{msec}$ (Meeren et al., 2002), signals were analyzed, as in earlier studies (Lüttjohann and van Luijtelaar, 2012; Lüttjohann et al., 2013), in time windows of $500 \mathrm{~ms}$, shifting along the transition periods in steps of $125 \mathrm{~ms}$. First, spectral decomposition was performed using a multi-taper approach (Percival and Walden, 1993 ) in the frequency range of 2 to $60 \mathrm{~Hz}$. Given the size of the analysis window, the frequency resolution was restricted to an accuracy of $2 \mathrm{~Hz}$. Next, frequency resolved Granger Causality (GC) was computed for all cortico-thalamic, thalamo-thalamic and intracortical (between different layers of the somatosensory cortex) channel pairs. We used nonparametric spectral factorization (Dhamala et al., 2008a,b), as implemented in FieldTrip (Oostenveld et al., 2011). The more traditional approach to compute frequency resolved GC is based on multivariate autoregressive models. The advantage of the non-parametric approach is that it does not require the estimation of such autoregressive models, and thus bypasses non-trivial signal processing issues such as the estimation of an optimal model order. GC quantifies the extent to which the prediction of signal A based on its own past can be improved by also including information of the past of signal B. In this way Granger Causality has become a well-established way to infer directional information flow between neuronal populations based on statistical dependencies between signals, and provides a complementary approach to the more traditional stimulation or ablation approach (Bressler and Seth, 2011). In the current experiment a causal influence or directional coupling was only inferred if GC values for the coupling direction A- > B were significantly higher than for coupling direction $\mathrm{B}->\mathrm{A}$ or the other way around. An increase (e.g. non-epileptic control vs. SWD) of GC of equal strength for both coupling directions ( $A->B$ and $B->A$ ) is regarded as an increase in 'bidirectional' coupling without a dominant, guiding structure.

To assure that an unequal increase in GC cannot be explained by a differential improvement of the signal to noise ratio (SNR), taking place with SWD occurrence, mean SNR improvements of the entire signal (not separated for all different $2 \mathrm{~Hz}$ frequency bins, which is a possible weakness of this study; this particularly applies to the high frequency results since they usually correspond to low amplitude signals compared to the total energy in the signal) were calculated for each channel by dividing the variance during SWD by the variance during non-epileptic control periods. SNR improvements were then compared by a one-way ANOVA, with SNR improvement as dependent variable and channel as between-subjects factor. On top of this for channel pairs showing directed connectivity (which might be explained by a stronger improvement in SNR in the 'leading' channel as compared to the 'lagging' channel) putative changes in SNRs were also evaluated with a more sensitive (one sided) paired t-test.

Statistical comparison of GC values between coupling directions (A- > B vs B- >A) was done with a non-parametric cluster based permutation test (Maris and Oostenveld, 2007). This test has been shown to be a reliable statistical method for the analysis of neurophysiological data requiring comparison along multiple time and frequency bins, with efficient control of type II errors (Maris et al., 2007). Basically the following steps are performed:

- For a given channel pair, and for every time-frequency point the difference in GC between the control and transition periods is quantified by means of a paired t-statistic, yielding maps of tvalues of the observed effect. 
- These t-maps are thresholded based on a t-value corresponding to uncorrected two-tailed p-value of 0.05 , and clusters are formed based on temporal and spectral adjacency.

- For each cluster the sum of the t-values is calculated, which serves as test statistic.

- A reference distribution of the test-statistic under the null hypothesis is generated using a permutation approach: the GC time-frequency maps in the control and transition periods are randomly distributed across two groups and for each randomization the test-statistic was recalculated as described above. Using a large number of randomizations, the resulting reference distribution was used to estimate the probability of observing the test-statistic under the null hypothesis. In case the test-statistic computed from the real data set is positioned below the 2.5-th or above the 97.5-th quantile of the reference distribution ( $\mathrm{p}<0.05$ two-sided), a cluster is considered as representing a significant difference between control and transition periods.

For the present analysis, 500 randomizations were used.

The same statistical test was used to compare GC values of

- pre-ictal - > ictal transition periods to non-epileptic control periods,

- ictal - > post-ictal transition periods to non-epileptic control periods and

- ictal - > post-ictal transition periods to ‘stable SWD' control periods.

All statistical and signal analyses were performed with FieldTrip, an open-source Matlab-based toolbox for advanced analysis of e.g. electrophysiological data (Oostenveld et al., 2011).

Only data from brain structures with a histological verified proper electrode position were included in the statistical analysis (Table 1). To facilitate the finding of the location of the tip of the recording electrodes, a direct current ( $9 \mathrm{~V}, 25 \mu \mathrm{A}, 10 \mathrm{sec}$ duration) was passed through each electrode in the deeply anaesthetized rat at the end of the experiment. Next, rats were perfused with a potassiumferrocyanide formaldehyde - phosphate solution, coloring these lesions at the end of each electrode tip. Brains were fixed in a $30 \%$ sucrose solution, $0.1 \mathrm{ml} \mathrm{PBS}$, cut in $40 \mu \mathrm{m}$ coronal slices with the aid of a microtome, and stained with Cresyl violet. Only electrodes for which the midpoint of the small lesion was located within the target structure were considered properly implanted and included in statistical analysis.

\section{Results}

Spike and wave discharges, electrophysiological observations and SNR

All rats showed SWDs, mean 10 per hour, mean duration SWDs 7 seconds (range from 6 to 11 seconds). Rhythmic spike and wave activity in the typical frequency of $7-11 \mathrm{~Hz}$ was present in all recorded

Table 1

Results of histological verification of electrode position.

\begin{tabular}{|c|c|c|c|c|c|c|c|c|}
\hline & Ctx4 & Ctx 5 & Ctx6 & ATN & Po & VPM & cRTN & rRTN \\
\hline Rat1 & $\mathrm{x}$ & $\mathrm{X}$ & $\mathrm{x}$ & $\mathrm{x}$ & $\mathrm{X}$ & $\mathrm{x}$ & $\mathrm{X}$ & $\mathrm{X}$ \\
\hline Rat2 & $x$ & $x$ & $\mathrm{x}$ & $x$ & $\mathrm{x}$ & & $x$ & \\
\hline Rat3 & $x$ & $x$ & $\mathrm{x}$ & $x$ & $\mathrm{x}$ & & $\mathrm{x}$ & $\mathrm{X}$ \\
\hline Rat4 & $\mathrm{x}$ & $\mathrm{x}$ & $\mathrm{x}$ & & $\mathrm{x}$ & $\mathrm{X}$ & $\mathrm{x}$ & \\
\hline Rat5 & $x$ & $\mathrm{x}$ & $\mathrm{x}$ & & $\mathrm{x}$ & $\mathrm{x}$ & $x$ & $\mathrm{x}$ \\
\hline Rat6 & $x$ & $\mathrm{x}$ & $\mathrm{x}$ & $\mathrm{X}$ & $\mathrm{x}$ & $\mathrm{x}$ & $x$ & $\mathrm{x}$ \\
\hline Rat7 & $x$ & $\mathrm{x}$ & $\mathrm{x}$ & $x$ & $\mathrm{x}$ & $\mathrm{x}$ & $\mathrm{x}$ & $\mathrm{x}$ \\
\hline Rat8 & $\mathrm{x}$ & $\mathrm{x}$ & $\mathrm{x}$ & $x$ & & $\mathrm{x}$ & & \\
\hline Rat9 & $x$ & $\mathrm{x}$ & $\mathrm{x}$ & $x$ & $\mathrm{X}$ & $\mathrm{x}$ & $\mathrm{X}$ & $\mathrm{x}$ \\
\hline Rat10 & $x$ & $\mathrm{x}$ & $\mathrm{x}$ & $\mathrm{x}$ & & $\mathrm{x}$ & & \\
\hline Rat11 & $\mathrm{x}$ & $x$ & $\mathrm{x}$ & $x$ & $\mathrm{X}$ & $\mathrm{x}$ & $\mathrm{X}$ & $\mathrm{x}$ \\
\hline Rat12 & $x$ & $x$ & $\mathrm{x}$ & $x$ & $\mathrm{x}$ & $\mathrm{x}$ & & $\mathrm{x}$ \\
\hline Rat13 & $\mathrm{X}$ & $\mathrm{X}$ & $\mathrm{X}$ & & $\mathrm{x}$ & & $\mathrm{X}$ & $\mathrm{x}$ \\
\hline Rat14 & $\mathrm{x}$ & $\mathrm{x}$ & $\mathrm{x}$ & $\mathrm{X}$ & $\mathrm{x}$ & $\mathrm{x}$ & $\mathrm{x}$ & \\
\hline Rat15 & $x$ & $\mathrm{x}$ & $\mathrm{x}$ & $x$ & $\mathrm{x}$ & & $\mathrm{x}$ & \\
\hline Rat16 & $\mathrm{x}$ & $\mathrm{x}$ & $\mathrm{x}$ & & $\mathrm{x}$ & $\mathrm{x}$ & $x$ & \\
\hline
\end{tabular}

$\mathrm{X}$ indicates correctly located electrode. channels. SWD-like activity could be seen by visual inspection earlier in the cortex than in the thalamus in $20 \%$ of the rats. In these rats the local start of SWD activity preceded the thalamic involvement by up to 1 second (see van Luijtelaar et al., 2011 for figure). In addition, in about $20 \%$ of rats SWD activity was found to terminate about 1 second earlier in the thalamic recordings as compared to the cortical recordings (see van Luijtelaar et al., 2011 for figure).

All channels showed an increased signal to noise ratio (SNR) during the SWD. ANOVA on SNR improvement revealed no significant main effect on recording channel $(p>0.05)$. All channels showed an improvement in SNR from non-epileptic to epileptic LFPs of equal strength. The same was revealed by the more sensitive one-sided t-tests, performed for channel pairs showing a 'directed connectivity' (see below) (all ps $>0.05$ ).

\section{Cortico-Thalamic changes in Granger Causality (GC) at SWD onset and SWD offset}

All cortico-thalamic pairs showed low GC (values between 0.01 and 0.05 ) during the non-epileptic control as well as during the pre-ictal period. During the complete SWD all channel pairs showed a significant increase in GC as compared to control (all ps $<0.025$ ), which was the case for both coupling directions (see Fig. 1 for an example as well as Fig. 4 and Table 2 for summary).

For most cortico-thalamic pairs (ctx6-VPM, ctx5-VPM, ctx6-Po, ctx4-Po, ctx6-ATN, ctx5-ATN, ctx5-cRTN) (Table 2) a higher GC was seen for the coupling direction cortex- $>$ thalamus as compared to coupling direction thalamus- $>$ cortex (all p-values $<0.025$ ), but this directional coupling was restricted to the onset (first $500 \mathrm{~ms}$ following FCTS) of the SWD (Fig. 2a). Whereas for channel pairs ctx6- > ATN, ctx5- > ATN, ctx5- > cRTN this increase was found for the higher $(20-40 \mathrm{~Hz})$ frequencies, for the other channel pairs (ctx6- > VPM, ctx5- > VPM, ctx6- > Po, ctx4- > Po) this increase was seen for the low $(4-12 \mathrm{~Hz})$ frequencies including the typical SWD frequency range of 8-10 Hz. Channel pair ctx4- > ATN showed higher GC values for the coupling direction cortex- > thalamus in the low frequencies, which was restricted to the onset of SWD. In addition, this channel pair (ctx4-ATN) also showed higher GC-values for the coupling direction cortex- $>$ thalamus in the frequency range of 20 $50 \mathrm{~Hz}$, which was not restricted to SWD onset but was seen persistently during the entire SWD. Channel pairs ctx4- > cRTN, and ctx4- > VPM showed a persistent (during the entire SWD) higher GC values for the coupling direction cortex- $>$ thalamus in the high frequencies (20$50 \mathrm{~Hz}$ ) (Fig. 2b).

Channel pair ctx4-rRTN showed a higher directional GC value for the cortex- > thalamus, which was persistently present throughout the entire SWDs in the $20-50 \mathrm{~Hz}$ frequency range. In addition, this was the only cortico-thalamic channel pair for which a persistent (not restricted to SWD onset) dominant drive from the cortex was also seen in the SWD frequency band 8-10 Hz. However, it only remained present until 1.5 second prior to SWD offset (Fig. 3a). The lack of a higher cortex- $>$ thalamus coupling during the last $1.5 \mathrm{sec}$ as compared to thalamus- > cortex coupling was due to a decrease of cortex- $>$ thalamus coupling rather than an increase in the thalamus- > cortex coupling. This was verified by significant difference between GC in SWD transition period as compared to stable SWDcontrol period which started to occur at the same time-point, whereas for all other channel pairs this only started to be significant at SWD offset.

Most cortico-thalamic channel pairs showed low (no significant difference as compared to non-epileptic control periods) GC values during the post-ictal period, similar to the pre-ictal and control period. The only two exceptions were channel pair ctx6-Po and ctx5-Po: they showed higher directional cortex- $>$ thalamus coupling 0.25 to $1.5 \mathrm{sec}$ following the offset of SWD (Fig. 3c). This higher cortex- $>$ thalamus coupling as compared to thalamo- > cortical coupling could be attributed to the 
Table 2

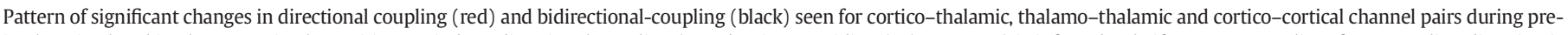

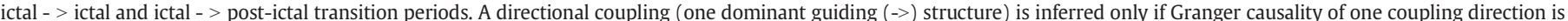

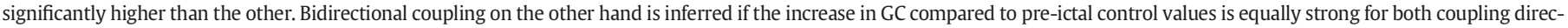
tions.

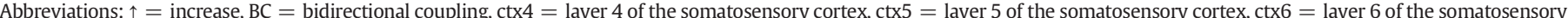

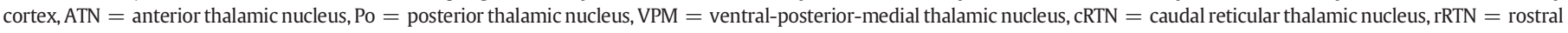
reticular thalamic nucleus, $\mathrm{C}=$ cortex, $\mathrm{T}=$ thalamus.

\begin{tabular}{|c|c|c|c|}
\hline & Channelpair & GC changes SWD onset & GC changes SWD offset \\
\hline \multirow{18}{*}{$\begin{array}{c}\text { C-T } \\
\& \\
\text { T-C }\end{array}$} & ctx5-Po & $\begin{array}{l}\uparrow \mathrm{BC}, 2-8 \mathrm{~Hz} \text {, persistent starting at } \\
\text { SWD onset }\end{array}$ & $\begin{array}{l}\uparrow \mathrm{BC}, 2-8 \mathrm{~Hz} \text {, persistent until SWD } \\
\text { offset } \\
\uparrow \mathrm{ctx5} 5>\mathrm{Po}, 2-8 \mathrm{~Hz}, 0.25 \text { to } 1.5 \mathrm{~s} \\
\text { following SWD offset }\end{array}$ \\
\hline & ctx6-Po & $\begin{array}{l}\text { } \text { ctx6->Po,4-12Hz, first 500ms } \\
\text { following SWD onset; } \\
\uparrow \mathrm{BC}, 4-12 \mathrm{~Hz} \text {, persistent starting } \\
\text { 500ms following SWD onset }\end{array}$ & $\begin{array}{l}\uparrow \mathrm{BC}, 4-12 \mathrm{~Hz} \text {, persistent until SWD } \\
\text { offset } \\
\uparrow \mathrm{ctx} 6->\mathrm{Po}, 2-8 \mathrm{~Hz}, 0.25 \text { to } 1.5 \mathrm{~s} \\
\text { following SWD offset }\end{array}$ \\
\hline & ctx4-Po & $\begin{array}{l}\uparrow \mathrm{ctx} 4->\mathrm{Po}, 8-20 \mathrm{~Hz} \text {, first } 500 \mathrm{~ms} \\
\text { following SWD onset; } \\
\uparrow \mathrm{BC}, 8-20 \mathrm{~Hz} \text {, persistent starting } \\
500 \mathrm{~ms} \text { following SWD onset }\end{array}$ & $\begin{array}{l}\uparrow \mathrm{BC}, 8-20 \mathrm{~Hz} \text {, persistent until SWD } \\
\text { offset }\end{array}$ \\
\hline & ctx6-VPM & $\begin{array}{l}\uparrow \mathrm{ctx6}->\mathrm{VPM}, 4-10 \mathrm{~Hz} \text {, first } 500 \mathrm{~ms} \\
\text { following SWD onset; } \\
\uparrow \mathrm{BC}, 4-10 \mathrm{~Hz} \text {, persistent starting } \\
\text { 500ms following SWD onset }\end{array}$ & $\begin{array}{l}\uparrow \mathrm{BC}, 4-10 \mathrm{~Hz} \text {, persistent until SWD } \\
\text { offset }\end{array}$ \\
\hline & ctx5-VPM & $\begin{array}{l}\uparrow \mathrm{ctx} 5-\mathrm{VPMM}, 4-10 \mathrm{~Hz} \text {, first } 500 \mathrm{~ms} \\
\text { following SWD onset; } \\
\uparrow \mathrm{BC}, 4-10 \mathrm{~Hz} \text {, persistent starting } \\
\text { 500ms following SWD onset }\end{array}$ & $\begin{array}{l}\uparrow \mathrm{BC}, 4-10 \mathrm{~Hz} \text {, persistent until SWD } \\
\text { offset }\end{array}$ \\
\hline & ctx6-ATN & $\begin{array}{l}\uparrow \mathrm{ctx6} 6 \mathrm{ATN}, 8-12 \mathrm{~Hz} \text { and } 25-35 \mathrm{~Hz}, \\
\text { first 500ms following SWD onset; } \\
\uparrow \mathrm{BC}, 8-12 \mathrm{~Hz} \text { and } 25-35 \mathrm{~Hz} \text {, persistent } \\
\text { starting 500ms following SWD onset }\end{array}$ & $\begin{array}{l}\uparrow B C, 8-12 \mathrm{~Hz} \text { and } 25-35 \text {, persistent } \\
\text { until SWD offset }\end{array}$ \\
\hline & ctx5-ATN & $\begin{array}{l}\text { } \text { ctx5->ATN, } 25-35 \mathrm{~Hz} \text {, first } \\
\text { 500ms following SWD onset; } \\
\uparrow \mathrm{BC}, 25-35 \mathrm{~Hz} \text {, persistent starting } \\
\text { 500ms following SWD onset }\end{array}$ & $\begin{array}{l}\uparrow B C, 20-30 \mathrm{~Hz} \text {, persistent until SWD } \\
\text { offset }\end{array}$ \\
\hline & ctx5-cRTN & $\begin{array}{l}\uparrow \mathrm{ctx} 5->\mathrm{CRTN}, 20-40 \mathrm{~Hz} \text {, first } 500 \mathrm{~ms} \\
\text { following SWD onset; } \\
\uparrow \mathrm{BC}, 20-40 \mathrm{~Hz} \text {, persistent starting } \\
\text { 500ms following SWD onset }\end{array}$ & $\begin{array}{l}\uparrow \mathrm{BC}, 20-40 \mathrm{~Hz} \text {, persistent until SWD } \\
\text { offset }\end{array}$ \\
\hline & ctx4-ATN & $\begin{array}{l}\text { Tctx4->ATN, 8-12 Hz, first 500ms } \\
\text { following SWD onset; }\end{array}$ & $\begin{array}{l}\uparrow \mathrm{ctx} 4->\mathrm{ATN}, 20-50 \mathrm{~Hz} \text {, persistent } \\
\text { until SWD offset }\end{array}$ \\
\hline & & & $\begin{array}{l}\uparrow B C, 8-12 \mathrm{~Hz} \text {, persistent until SWD } \\
\text { offset }\end{array}$ \\
\hline & & $\begin{array}{l}\uparrow \mathrm{BC}, 8-12 \mathrm{~Hz} \text {, persistent starting } \\
500 \mathrm{~ms} \text { following SWD onset }\end{array}$ & \\
\hline & ctx4-cRTN & $\begin{array}{l}\begin{array}{l}\text { Tctx4->CRTN } 20-50 \mathrm{~Hz} \text { persistent } \\
\text { starting at SWD onset }\end{array} \\
\text { ? }\end{array}$ & 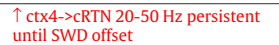 \\
\hline & ctx4-VPM & $\begin{array}{l}\uparrow c t x 4->V P M, 40-48 \mathrm{~Hz} \text {, persistent } \\
\text { starting at SWD onset }\end{array}$ & $\begin{array}{l}\uparrow c t x 4->V P M, 40-48 \mathrm{~Hz} \text {, persistent } \\
\text { until SWD ofset }\end{array}$ \\
\hline & ctx4-rRTN & $\begin{array}{l}\text { 个ctx4->rRTN, } 8-10 \mathrm{~Hz} \text { and } 20-50 \mathrm{~Hz} \\
\text { persistent starting at SWD onset }\end{array}$ & $\begin{array}{l}\uparrow c t x 4->r \text { RTN, } 8 \text {-10 Hz persistent } \\
\text { until } 1.5 \mathrm{~s} \text { before SWD offset; }\end{array}$ \\
\hline & & & $\begin{array}{l}\uparrow c t x 4->r \text { RTN, } 20-50 \mathrm{~Hz} \text { persistent } \\
\text { until SWD offset }\end{array}$ \\
\hline & ctx6-cRTN & $\begin{array}{l}\uparrow \mathrm{BC}, 6-30 \mathrm{~Hz} \text {, persistent starting at } \\
\text { SWD onset }\end{array}$ & $\begin{array}{l}\uparrow \mathrm{BC}, 6-30 \mathrm{~Hz} \text {, persistent until SWD } \\
\text { offset }\end{array}$ \\
\hline & ctx5-rRTN & $\begin{array}{l}\uparrow \mathrm{BC}, 6-40 \mathrm{~Hz} \text {, persistent starting at } \\
\text { SWD onset }\end{array}$ & $\begin{array}{l}\uparrow \mathrm{BC}, 6-40 \mathrm{~Hz} \text {, persistent until SWD } \\
\text { offset }\end{array}$ \\
\hline & ctx6-rRTN & $\begin{array}{l}\uparrow \mathrm{BC}, 6-12 \mathrm{~Hz} \text {, persistent starting at } \\
\text { SWD onset }\end{array}$ & $\begin{array}{l}\begin{array}{l}\uparrow \mathrm{BC}, 6-10 \mathrm{~Hz} \text {, persistent until SWD } \\
\text { offset }\end{array}\end{array}$ \\
\hline \multirow{10}{*}{ T-T } & cRTN-rRTN & $\begin{array}{l}\uparrow B C, 4-14 \mathrm{~Hz} \text {, persistent starting at } \\
\text { SWD onset }\end{array}$ & $\begin{array}{l}\uparrow \mathrm{BC}, 4-14 \mathrm{~Hz} \text {, persistent persistent } \\
\text { until } 1 \mathrm{~s} \text { prior to SWD offset } \\
\uparrow \mathrm{cRTN}->\mathrm{RTN}, 4-14 \mathrm{~Hz}, 1 \mathrm{~s} \text { prior to } \\
\text { SWD offset until } 0.25 \text { following SWD } \\
\text { offset }\end{array}$ \\
\hline & cRTN-Po & $\begin{array}{l}\uparrow \text { cRTN->Po, 6-12 Hz persistent } \\
\text { starting at SWD onset }\end{array}$ & $\begin{array}{l}\text { TcRTN->Po, 4-12 Hz persistent until } \\
\text { SWD offset }\end{array}$ \\
\hline & rRTN-Po & $\begin{array}{l}\uparrow \text { rRTN->Po 6-10Hz persistent } \\
\text { starting at SWD onset }\end{array}$ & $\begin{array}{l}\text { 个rRTN->Po } 6-10 \mathrm{~Hz} \text { persistent until } \\
\text { SWD offset }\end{array}$ \\
\hline & ATN-Po & $\begin{array}{l}\uparrow \text { ATN->Po 4-12 Hz persistent } \\
\text { starting at SWD onset }\end{array}$ & $\begin{array}{l}\uparrow \text { ATN->Po } 4-12 \mathrm{~Hz} \text { persistent until } \\
\text { SWD offset }\end{array}$ \\
\hline & cRTN-VPM & $\begin{array}{l}\uparrow c R T N->V P M \\
\text { 4 }-12 \mathrm{~Hz} \text { persistent } \\
\text { starting at SWD onset }\end{array}$ & $\begin{array}{l}\uparrow c \text { RTN->VPM 4-12 Hz persistent } \\
\text { until SWD offset }\end{array}$ \\
\hline & VPM-Po & $\begin{array}{l}\uparrow \text { VPM->Po } 25-45 \mathrm{~Hz} \text {, first } 500 \mathrm{~ms} \\
\text { following SWD onset; } \\
\uparrow \text { BC, } 25-45 \mathrm{~Hz} \text {, persistent starting } \\
\text { 500ms following SWD onset }\end{array}$ & $\begin{array}{l}\uparrow \mathrm{BC}, 25-45 \mathrm{~Hz} \text {, persistent until SWD } \\
\text { offset }\end{array}$ \\
\hline & VPM-ATN & $\begin{array}{l}\uparrow \text { VPM->ATN } 30-35 \mathrm{~Hz} \text {, first } 500 \mathrm{~ms} \\
\text { following SWD onset; } \\
\uparrow \mathrm{BC}, 30-35 \mathrm{~Hz} \text {, persistent starting } \\
\text { 500ms following SWD onset }\end{array}$ & $\begin{array}{l}\uparrow B C, 30-35 \mathrm{~Hz} \text {, persistent until SWD } \\
\text { offset }\end{array}$ \\
\hline & rRTN-ATN & $\begin{array}{l}\uparrow \mathrm{BC}, 2-4 \mathrm{~Hz} \text {, persistent starting at } \\
\text { SWD onset }\end{array}$ & $\begin{array}{l}\uparrow \mathrm{BC}, 2-4 \mathrm{~Hz} \text {, persistent until SWD } \\
\text { offset }\end{array}$ \\
\hline & cRTN-ATN & $\begin{array}{l}\uparrow \mathrm{BC}, 2-20 \mathrm{~Hz} \text {, persistent starting at } \\
\text { SWD onset }\end{array}$ & $\begin{array}{l}\uparrow \mathrm{BC}, 2-20 \mathrm{~Hz} \text {, persistent until SWD } \\
\text { offset }\end{array}$ \\
\hline & rRTN-VPM & $\begin{array}{l}\uparrow \mathrm{BC}, 4-14 \mathrm{~Hz} \text {, persistent starting at } \\
\text { SWD onset }\end{array}$ & $\begin{array}{l}\begin{array}{l}\uparrow B C, 4-14 \mathrm{~Hz} \text {, persistent until SWD } \\
\text { offset }\end{array}\end{array}$ \\
\hline \multirow{3}{*}{ C-C } & ctx4-ctx5 & $\begin{array}{l}\uparrow \mathrm{BC}, 6-40 \mathrm{~Hz} \text {, persistent starting at } \\
\text { SWD onset }\end{array}$ & 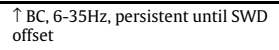 \\
\hline & ctx4-ctx6 & $\begin{array}{l}\uparrow \mathrm{BC}, 2-20 \mathrm{~Hz} \text {, persistent starting } \\
1.5 \mathrm{~s} \text { before SWD onset } \\
\uparrow \mathrm{BC}, 20-50 \mathrm{~Hz} \text {, persistent starting } \\
\text { at } \mathrm{SWD} \text { onset }\end{array}$ & $\begin{array}{l}\uparrow B C, 2-50 \mathrm{~Hz} \text {, persistent until SWD } \\
\text { offset }\end{array}$ \\
\hline & ctx5-ctx6 & $\begin{array}{l}\uparrow \mathrm{BC}, 2-20 \mathrm{~Hz} \text {, persistent starting } 1 \mathrm{~s} \\
\text { before SWD onset } \\
\uparrow \mathrm{BC}, 20-40 \mathrm{~Hz} \text {, persistent starting at } \\
\text { SWD onset }\end{array}$ & $\begin{array}{l}\uparrow \mathrm{BC}, 4-40 \mathrm{~Hz} \text {, persistent until SWD } \\
\text { offset }\end{array}$ \\
\hline
\end{tabular}


A

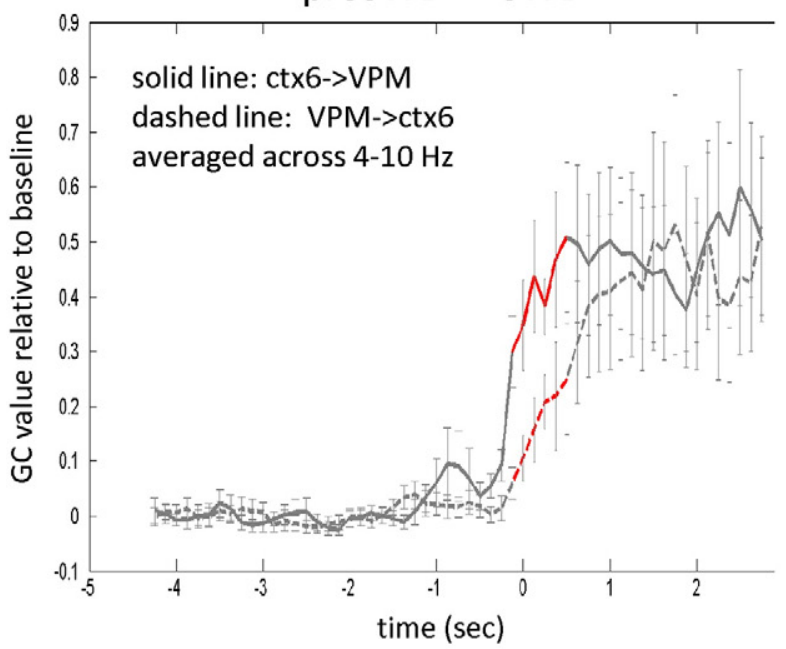

B

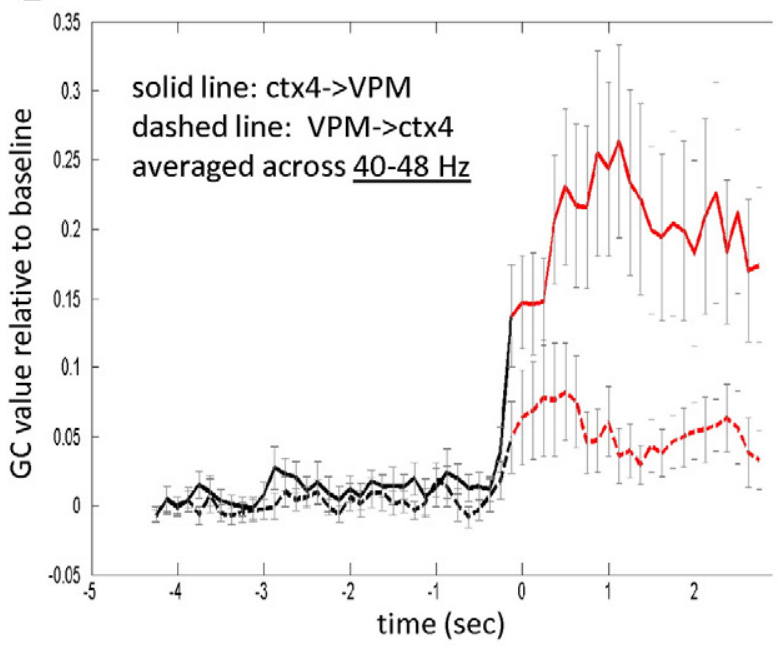

C

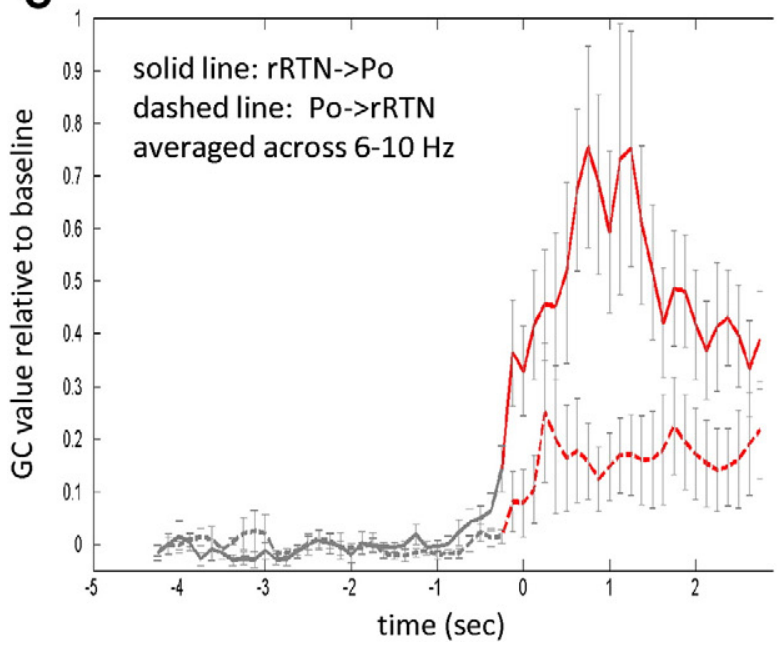

SWD -> postSWD
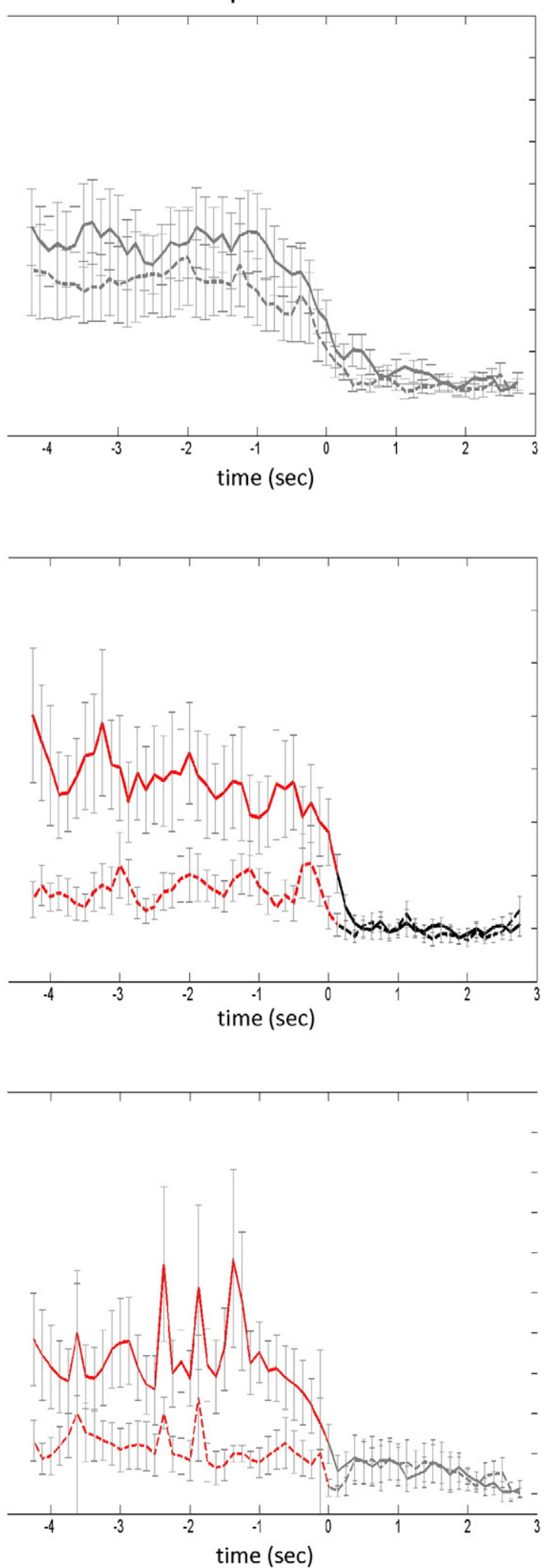

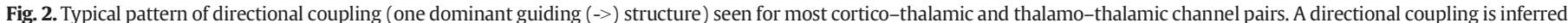

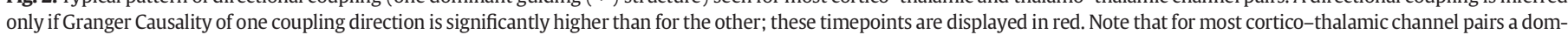

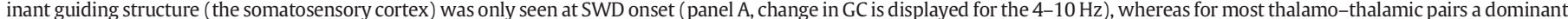

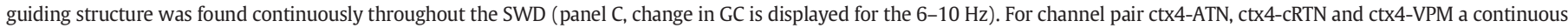

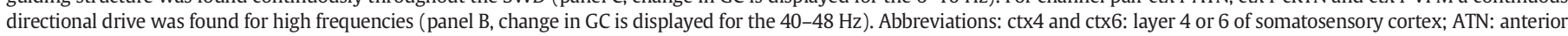
thalamic nucleus; VPM: ventral-postero-medial thalamic nucleus; rRTN: rostral reticular thalamic nucleus. 
A

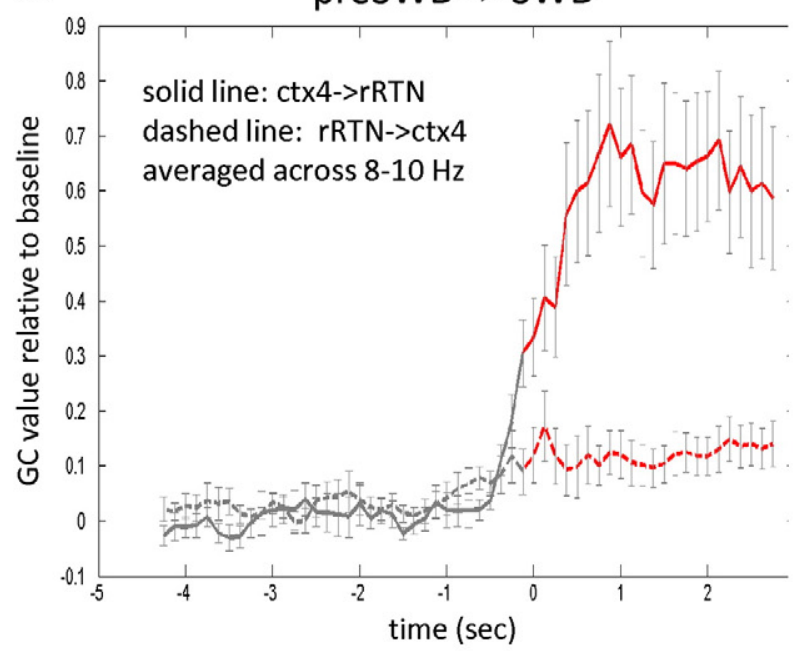

B

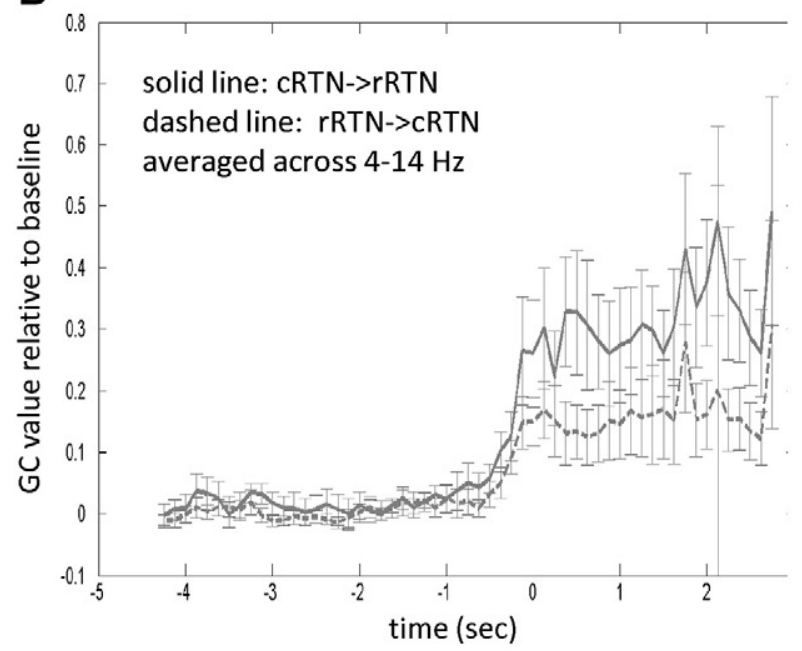

C

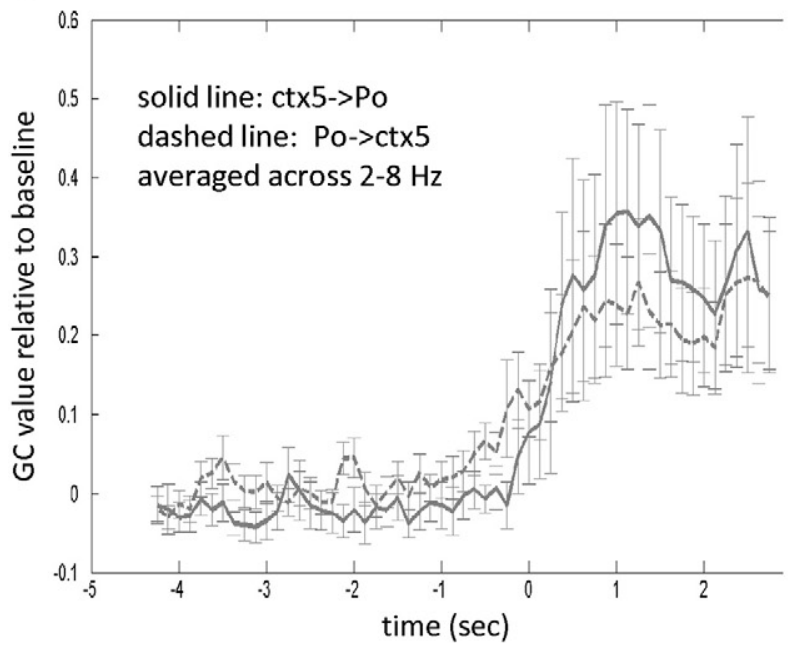

SWD -> postSWD
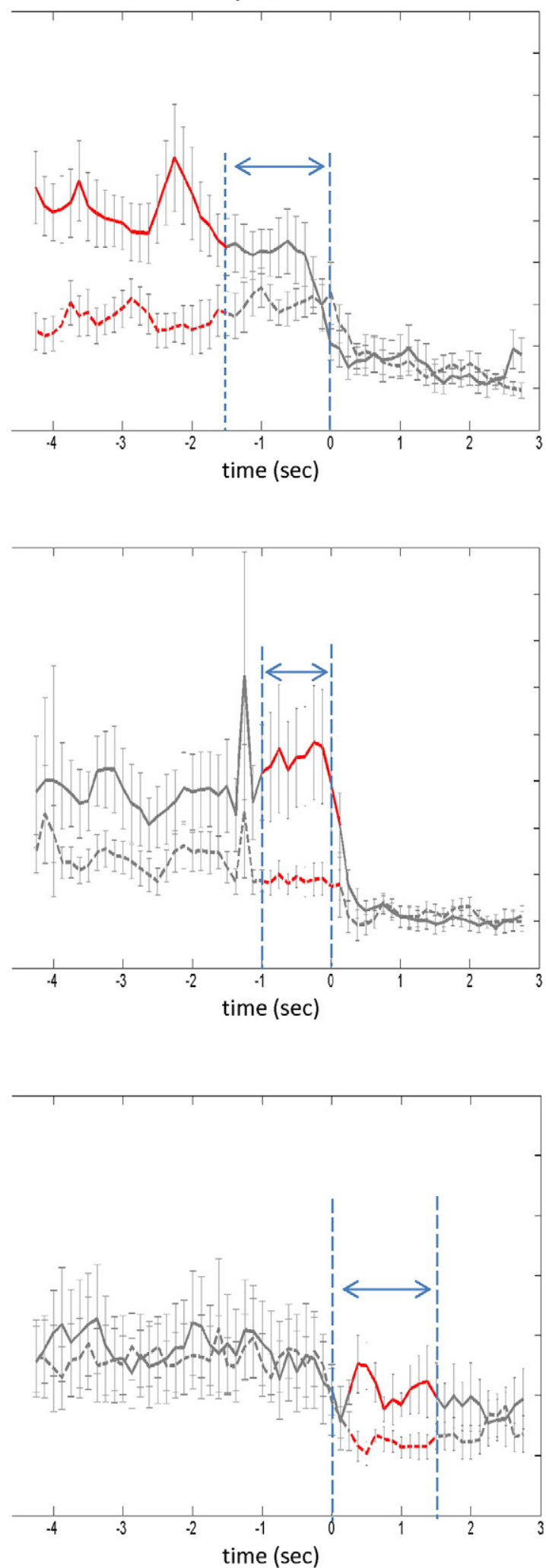

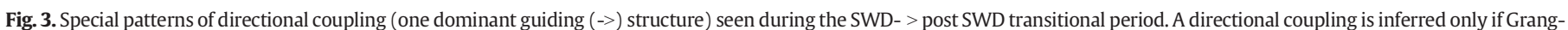

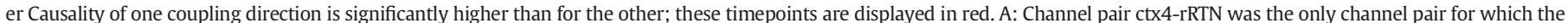

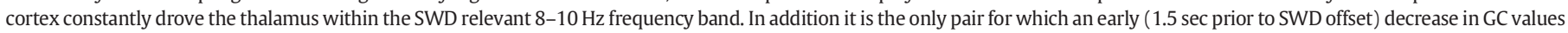

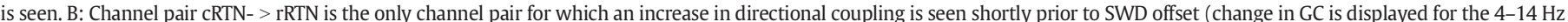

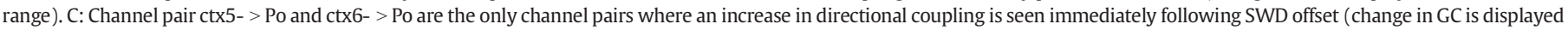

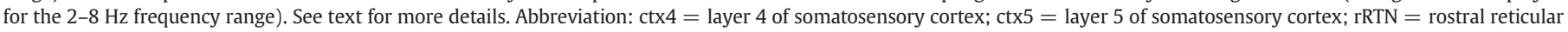
thalamic nucleus; $\mathrm{Po}=$ posterior thalamic nucleus. 
pre LCTS

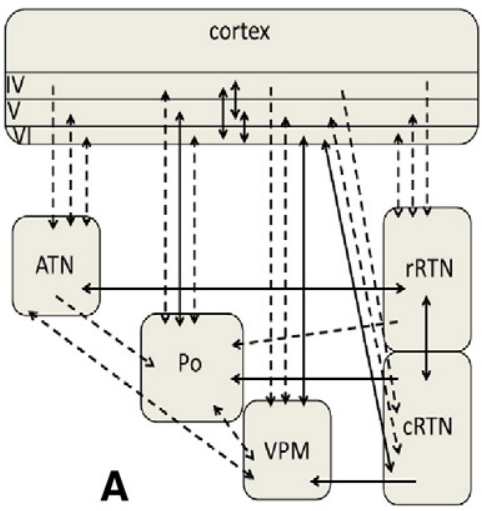

pre LCTS

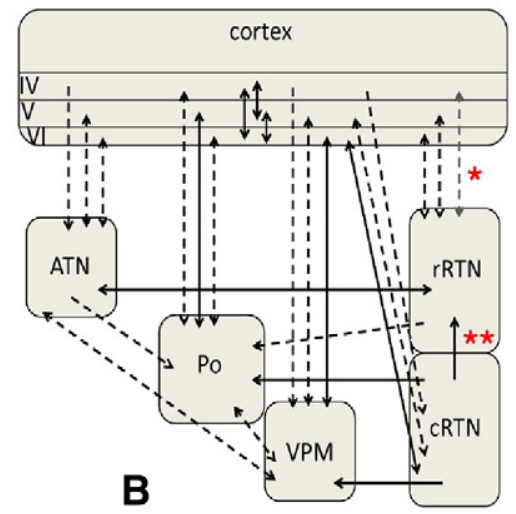

post LCTS

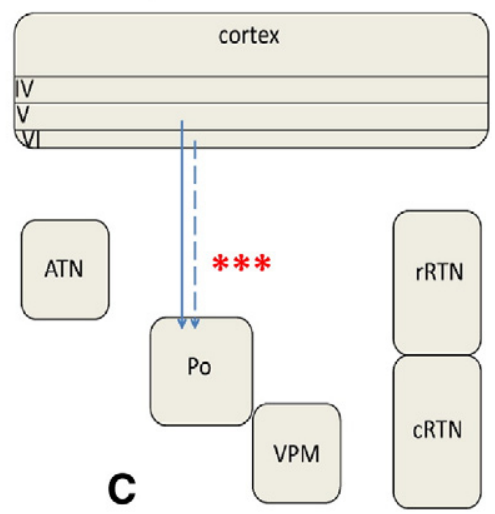

\section{* $1.5 \mathrm{sec}$ prior to LCTS ${ }^{* *} 1 \mathrm{sec}$ prior to LCTS ${ }^{* * *}$ till $1.5 \mathrm{sec}$ following LCTS}

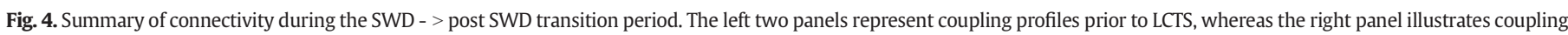

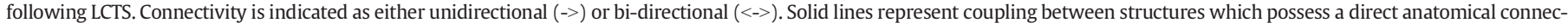

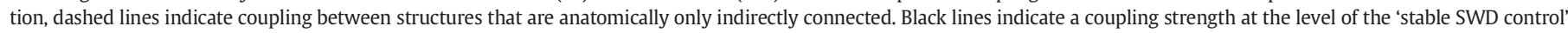

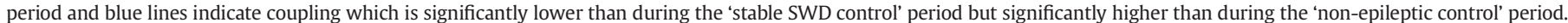

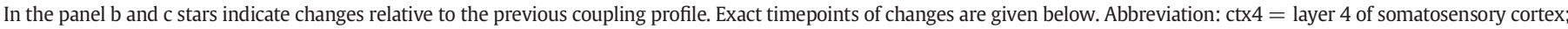

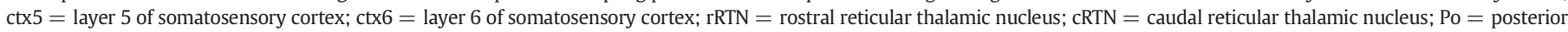
thalamic nucleus; VPM = ventral posteromedial thalamic nucleus; ATN = anterior thalamic nucleus; LCTS = last cortico-thalamo-cortical spike of SWD.

fact that the thalamo-cortical coupling decreased to baseline values following the SWD offset, whereas the cortex- $>$ thalamus coupling remained on an increased GC level. It can be noted that the latter channel pair (ctx5-Po) is not only special regarding its GC pattern in the post ictal period. In contrast to most channel pairs (as described above) ctx5Po does not show a higher cortex- $>$ thalamus directional coupling as compared to thalamus- > cortex coupling at SWD onset, but GC coupling for both directions was equally strong (bidirectional).

Thalamo-Thalamic changes in Granger Causality at SWD onset and SWD offset

Thalamo-Thalamic GC values during the non-epileptic control as well as during the pre-ictal period were low (0.01 to 0.05$)$. In contrast, GC values during SWD were increased compared to the non-epileptic control period for both coupling directions (all ps < 0.025) (see Fig. 4 and Table 2 for summary of results). For most channel pairs a dominant coupling direction (stronger increase in GC for one direction compared to the other) could be identified during SWD (Table 2). While most of the cortico-thalamic channel pairs revealed this directed or dominant coupling direction only at SWD onset (first $500 \mathrm{~ms}$ ), this restriction to SWD onset was seen for only two intra-thalamic pairs: VPM- > Po and VPM- > ATN.

In contrast most thalamo-thalamic channel pairs showed persistent directional coupling throughout the whole SWD. This was observed for the following pairs (arrow indicates the direction of interaction): cRTN- > VPM, cRTN- > Po, rRTN- > Po, ATN- > Po. In all these cases dominant coupling was seen in the frequency band between $4-12 \mathrm{~Hz}$, including the major SWD frequency range (Fig. 2c).

An exception was found for channel pair cRTN-rRTN: the SWD related increase in GC was equally strong for both coupling directions at SWD onset and during SWD, while a directional dominance was found to occur $1 \mathrm{sec}$ prior to SWD offset until $0.2 \mathrm{sec}$ following its offset. In this period significantly higher GC values were found for the direction cRTN- > rRTN in the 4-14 Hz frequency range (Fig. 3b). This dominance was attributed to an increase in GC for CRTN-rRTN towards the end of the SWD as well as a slight decrease of rRTN- > cRTN GC. It can be noted that the time-point of change in coupling dominance is immediately following the earlier described decrease in GC seen for the ctx4-
$>$ rRTN and immediately prior to the increase noted for ctx5-Po and ctx6- > Po (see Fig. 3 for temporal preceding at SWD offset).

\section{Cortico-Cortical changes in Granger Causality at SWD onset and SWD offset}

All intra-cortical channel pairs showed a strongly increased bidirectional coupling (equally strong increases in GC for direction A- > B as B- > A) during SWD as compared to control periods (all p $<0.025$ ) (see Fig. 4 and Table 2 for summary of results). Interestingly, whereas for channel pair ctx4-ctx5 this increase was only seen to start at FCTS, channel pair ctx4-ctx6 and ctx6-ctx5 already showed a significant increase in coupling as early as 1.5 and 1 second prior to FCTS, respectively. Following SWD offset (LCTS) GC values retuned to baseline values for all cortico-cortical channel pairs.

\section{Discussion}

The study aimed to investigate dynamics of cortico-thalamocortical network interactions, which are seen during the initiation and termination of SWD, using frequency resolved Granger Causality, quantifying directional coupling between cortex and different thalamic regions. In the current study directional coupling was inferred only if GC values for one coupling direction (e.g. A- > B) was significantly higher than for the other coupling direction (B- $>A$ ). To exclude the risk that such an unequal change in $\mathrm{GC}$ is the result of an unequal change in the SNR, SNR improvements seen with SWD occurrence were statistically compared between channels, although in a nonfrequency resolved manner. Since no difference in SNR improvement was detected between channels it can firmly be assumed that such unequal increases in GC between directions are not the result of unequal improvements in SNR between channels. Rather it can safely be assumed/concluded to represent true directional coupling.

An increase in GC (e.g. SWD vs. control) of equal strength for both coupling directions ( $\mathrm{A}->\mathrm{B}$ and $\mathrm{B}->\mathrm{A}$ ) was regarded as an increase in 'bidirectional' coupling without a dominant, guiding structure.

To achieve an extended picture of network changes associated with SWD initiation and termination, signals were obtained from multiple thalamic sites, proposed to play an important role in SWD generation, 
as well as from the deep layers of the somatosensory cortex, the proposed SWD instigator zone.

Pre-ical intra-cortical network changes and a 'driving' cortex at SWD onset

The generation of SWD was characterized by an early, pre-ictal (1.5 second prior to SWD onset) increase in bidirectional coupling between intracortical layers of the somatosensory cortex as well as a strong increase in directional coupling (higher increase of GC for coupling direction cortex- $>$ thalamus as compared to thalamus- > cortex) from the somatosensory cortex to most but not all thalamic nuclei seen during the first $500 \mathrm{~ms}$ of SWD.

The majority of the coupling results including their directions and dynamics are in strong concordance with earlier results on the dynamics of network interactions for the generation of SWD using a different, 'directed' connectivity analysis (non-linear association analysis) (Lüttjohann and van Luijtelaar, 2012; Meeren et al., 2002).

The early, pre-ictal increase in intracortical coupling might be the reflection of a strong pre-ictal increase in neuronal spiking in the deep layers ( $\mathrm{V}$ and $\mathrm{VI}$ ) of the somatosensory cortex as reported in GAERS rats (Polack et al., 2007). Likewise early pre-ictal increase in spectral delta and theta power were described for the deep layers of the somatosensory cortex of WAG/Rij rats (Lüttjohann et al., 2013). All might represent a 'preparation state for SWD initiation' performed by an epileptic focus, proposed to be located in the deep somatosensory cortex by the cortical focus theory on SWD generation (Meeren et al., 2002, 2005; Polack et al., 2007). It remains to be investigated, however, whether part of the pre-ictal connectivity changes are attributable to changes in the level of vigilance. Given the channel pair selectivity of these pre-ictal changes, this is unlikely, however, since vigilance related changes can be expected to be of a more global nature.

Also the directional drive from cortex to most thalamic nuclei seen at the onset (first $500 \mathrm{~ms}$ ) of SWD is in line with the cortical focus theory.

Sitnikova et al. (2008), also using Granger Causality to study changes directional coupling at SWD onset, by contrast did not find a dominant directional drive between the frontal cortex and the thalamus (VPM) at SWD onset. On the other hand, David et al. (2008) applying GC on fMRI data of GAERS rats did find a cortical drive at SWD onset between the somatosensory cortex and thalamus as well as between somatosensory cortex and striatum. Together it seems that a dominant cortical drive can only be revealed for the somatosensory cortex but not for other cortical sites, which is in good agreement with the cortical focus theory (Meeren et al., 2002, 2005; Polack et al., 2007).

For some cortico-thalamic channel pairs the directed cortical drive was found in high frequencies $(20-40 \mathrm{~Hz}$ ) above the SWD characteristic $8-12 \mathrm{~Hz}$ band. Given the non-sinusoidal nature of SWD and a relatively low power of the signal in such high frequencies, these results need to be interpreted with great caution. Morphological differences of the spikes in the different brain structures may explain part of our findings. The spectral representation of a spike waveform necessarily contains multiple frequency components, and thus also spreads out to a frequency range that is higher than the $8-12 \mathrm{~Hz}$ band. Yet, it cannot be excluded that the high frequency results reflect true neuronal oscillatory activity. The neocortex contains different types of cells, such as the fast-rhythmic bursting neurons and fast spiking neurons which are capable to fire in a high frequent manner or in spike burst recurring at 30-50 Hz (Steriade, 2003). Furthermore, changes in GABAergic and Glutamatergic neurotransmission have been found to induce changes in spectral power of the EEG in these frequencies (Coenen and van Luijtelaar, 1989; Halonen et al., 1992; Lally et al., 2014). It remains therefore possible that different combinations of oscillation cells, each with their own firing and recurrent rates, permit each brain region to affect other parts of the brain within a unique combination of frequencies. This suggestion, however, requires additional experimental verification, for example with the aid of single unit recordings.
Most intrathalamic pairs showed a persistent (throughout the complete SWD) drive in SWD frequencies, whereas such a coupling pattern was only found for one cortico-thalamic channel pair. Interestingly, in all except one (cRTN-VPM) of these cases the Po was driven by another thalamic nucleus. This is in line with earlier network analytical studies, which associated the Po as having a role of synchronizing and channeling thalamic output to the cortex (Lüttjohann and van Luijtelaar, 2012; Lüttjohann et al., 2013). The persistent drive of the cRTN to the VPM is in agreement with classical neurophysiological and computational data, reporting a tonic hyperpolarization of the thalamic relay cells during SWD, which is brought about by the GABAergic influence (Destexhe, 1999; Huguenard and McCormick, 2007; Steriade, 2003).

In any study correlating monopolarly derived signals one has to be aware of the risk of volume conduction. The assumed influence of it on our results, however, can be estimated to be small: Granger Causality is a measure that explicitly estimates the interaction between signals that is non-instantaneous in nature whereas volume conduction per definition is an instantaneous phenomenon. Yet, an increased instantaneous common contribution through volume conduction (or through physiological common input) may still lead to increased GC values. Importantly, however, an asymmetry in the change of estimated Granger Causality that is solely caused by volume conduction should be accompanied by an asymmetric change in signal power and as a consequence of an asymmetric change in SNR, which was statistically shown not to be the case in the current study.

\section{SWD termination}

Comparatively few studies have investigated the spontaneous termination of SWD, which was the major focus of the current study. Connectivity results on SWD termination are graphically summarized in Fig. 4. The most important outcomes of this part of the study are the following: 1) Whereas most channel pairs showed an abrupt return from high GC values during SWDs to low non-epileptic control GC values at LCTS, channel pair ctx4-rRTN showed a more gradual decrease of its directional coupling (from layer 4 of the somatosensory cortex to the rostral RTN). It started as early as 1.5 seconds prior to LCTS (compare Figs. 4a and b). 2) This was followed by an increase in directional coupling from the caudal RTN to the rostral RTN; it started at about 1 second prior to LCTS and lasted until 0.25 seconds following it (compare Figs. 4a and b). 3) A directional coupling from cortical layer 5 and 6 to the Po was noticed: it lasted from 0.25 until $1.5 \mathrm{sec}$ following LCTS (Fig. 4c). This was due to the continuation of the increased SWD GC in cortex-thalamus direction whereas GC in the opposite direction already returned to non-epileptic control levels at LCTS.

\section{Interpretation of result 1 and 2}

Since both findings occur in close temporal proximity to the offset of SWD they may be related to SWD termination. At the same time, since these findings occur relatively long (1.5 and 1 seconds) prior to the LCTS, it may be justifiable to conclude that the termination of SWD is a gradual process, which is already initiated 1.5 seconds prior to SWD offset. In addition, these results show that there is not one 'single' structure that 'takes the initiative' in SWD termination, but suggest that SWD termination relies on a combination of both cortico-thalamic as well as intrathalamic processes. Given the descriptive nature of signal analytical results as presented in the current study (Granger Causality is a wellestablished way to infer directional information flow between neuronal populations based on statistical dependencies between signals, and provides a complementary approach to the more traditional stimulation or ablation approach (Bressler and Seth, 2011)), the causal nature of both changes for SWD termination remains to be verified by experimental manipulations.

Changes in the intra-RTN communication were also related to the control of SWD duration based on the outcomes of two pharmacological experiments (Proulx et al., 2006; Sohal et al., 2003). Interestingly, 
whereas Sohal et al. (2003) achieved a shortening of SWD duration by enhancing intra-RTN communication via the local administration of the benzodiazepine clonazepam, Proulx et al. (2006) achieved a reduction of SWD duration via the decrease of intra-RTN communication by blocking gap-junctions. Others reported that the RTN of absence epileptic WAG/Rij rats is characterized by changes in $\mathrm{GABA}_{\mathrm{A}}$ receptor expression (a specific loss of $\alpha 3$ subunit immunoreactivity at inhibitory synapses) as compared to non-epileptic control rats (Liu et al., 2007). The outcomes of the present study now demonstrate for the first time the involvement of intra RTN communication in the control of SWD duration (SWD termination) in the natural context of spontaneously terminating (without pharmacological manipulations) SWD, in an in vivo genetic rat model under physiological circumstances. In addition, our study clarifies the nature of intra RTN changes towards the spontaneous termination of SWDs showing that an increase in coupling might need to occur from caudal to rostral RTN, whereas a decrease in coupling might need to occur in the direction from rostral to caudal RTN.

An early, gradual decrease (shortly after SWD onset) in GC was also reported between frontal cortex and VPM in the Sitnikova et al. (2008) study. Such a gradual decrease in cortex- $>$ thalamus coupling might be related to the gradual slowing of SWD frequencies seen in wavelet and other time-frequency analyses of SWD in patients and rats (Bosnyakova et al., 2006, 2007), which seem ultimately to lead to the termination of SWD. In longer lasting SWD, a repetition of this frequency modulation can be observed, which would also anticipate a waxing and waning pattern of the cortex- > thalamus GC coupling strength. Such a pattern, however might be obscured by the averaging across seizures, as was performed in this study.

It remains to be established, whether the early decrease between frontal cortex and VPM reported by Sitnikova et al. and the decrease between ctx 4 - > rRTN found in the current study are of equal relevance for the termination of SWD. As noted above ctx $4->$ rRTN was the only cortico-thalamic pair in this study, for which a persistent (from FCTS until $1.5 \mathrm{sec}$ prior to LCTS) dominant cortical drive was found in the SWD relevant $8-10 \mathrm{~Hz}$ frequency range. Such a consistent drive might be relevant for the maintenance of SWD, while its termination at $1.5 \mathrm{sec}$ prior to LCTS initiates SWD termination.

It needs to be mentioned, however, that no direct anatomic connections exist between the somatosensory cortex and rostral RTN. Therefore, it cannot be excluded that also a third structure, like e.g. the intralaminar nuclei, that have been proposed to play a role in SWD maintenance (Gorji et al., 2011; Seidenbecher and Pape, 2001) and which are known to receive and send input from the somatosensory cortex as well as to project to the rostral RTN (Cornwall et al., 1990; Kaufman and Rosenquist, 1985), is involved in this maintenance and termination process. In this way the intralaminar nuclei might either function as an additional, non-recorded relay station (ctx4 driving $(->)$ intralaminar nuclei - > rostral RTN), or as a common input to both structures that drives both with a different time delay (intralaminar nuclei - > ctx4 with signal shorter signal transduction time than intralaminar nuclei - > rostral RTN). The same might hold for other (non-directly) connected structures shown as dashed lines in Fig. 4.

In our study it can be noted that in both possible seizure termination processes (decrease in directional coupling ctx4- > rostral RTN and increase in directional coupling caudal RTN- > rostral RTN) the rostral RTN is involved: a reduction of cortical excitatory drive might result in a reduced oscillatory activity of the RTN. The increase of GABAergic coupling from caudal RTN onto the rostral RTN might have the same effect. Two more conclusions might be justified: a) the rostral RTN might function as an important resonator, which is of crucial relevance for the maintenance of SWD; and b) rostral and caudal RTN seem to have opposite effects on SWD, whereas increased oscillatory activity of the rostral RTN facilitates long SWDs, the caudal RTN rather functions as a break and increased activity of the caudal RTN hampers long lasting SWD.
These views are supported by earlier studies: Electro-microscopic inspection of the RTN by Liu and Jones (1999) revealed differences in receptive fields between RTN subparts (rostral and the more caudal ventral lateral RTN), and pharmacological and lesion studies revealed that a lesioning/inhibition of the caudal RTN led to an increase in SWD activity (Aker et al., 2006; Meeren et al., 2009), whereas a lesioning/ inhibiting of the oscillator rostral RTN resulted in a decrease of SWD activity (Aker et al., 2006; Berdiev and van Luijtelaar, 2009; Meeren et al., 2009).

\section{Interpretation of result 3}

Since the occurrence of a directional drive from cortical layer 5 and 6 to the Po is seen immediately after SWD termination it seems not to be related to the termination process itself any more. Rather, the increased deep somatosensory cortex- $>$ Po coupling, which remains present until $1.5 \mathrm{sec}$ following LCTS, seems to indicate that the epileptic focus, seated in the deep layers of the somatosensory cortex, tries to re-initiate the SWD.

Such a re-initiation attempt of the somatosensory cortex might be in accordance with signal analytical results (Maris et al. (2006), Bouwman et al. (2007)) showing that the chance of getting a new SWD is highest immediately after the previous one. In addition, it seems also to be in line with the above mentioned repetition of SWD frequency modulation that has been found for WAG/Rij rats and in patients during long lasting SWDs (Bosnyakova et al., 2006, 2007).

Interestingly, this enhanced cortico- $>$ thalamic coupling is maintained (for another $1.5 \mathrm{sec}$ following the SWD) with the Po. This nucleus has been proposed to play an important role in SWD initiation (Lüttjohann and van Luijtelaar, 2012). In a network-analytical study investigating the onset of SWD the Po showed highest increases in coupling with layer 5 of the somatosensory cortex, as established with the aid of a non-linear association analysis and was the only nucleus recorded in this study that showed a bidirectional-crosstalk to the cortex even within the first $500 \mathrm{~ms}$. In this way the Po was the only thalamic nucleus that forms a reverberation circuit with the cortex, which is regarded to be crucial for SWD generation and maintenance (Lüttjohann and van Luijtelaar, 2012); Huguenard and McCormick, 2007).

Channel pair ctx5-Po showed a strong bidirectional (equally strong increase GC for both coupling directions) coupling also in the current study, but also for three other channel pairs such a bidirectional coupling was seen. It needs to be mentioned however that, in contrast to the directional drive indicated by a unequal increase in GC for coupling direction A- > B vs. B- > A, shown not to be explainable by unequal changes in SNR between channels, an equally strong increase in GC for both coupling directions might still be related to an improvement of the signal to noise ratio seen during SWD. Therefore the latter GC results on bidirectional coupling are only reluctantly interpreted by us.

Following SWD termination, only the deep somatosensory cortex kept its increased coupling towards the Po, whereas the Po- > deep somatosensory cortex coupling stopped at LCTS. In this case no 'closed loop' reverberation circuit between the cortical, epileptic instigator zone and the Po is provided, which might explain that the SWD cannot be re-initiated. In the same line of reasoning a bidirectional coupling between somatosensory cortex and Po is expected at frequency modulation repetition points seen for longer lasting SWD (see above) where a termination of the SWD is prevented or a re-initiation succeeds. More direct measures of neuronal activity like multiple or single cell recordings in Po and deep cortical layers might help to verify these suggestions.

In summary,

- Frequency derived GC cortico-thalamic and intrathalamic network analyses showed that the onset of SWD (first $500 \mathrm{~ms}$ ) is characterized by a strong directional drive of the deep somatosensory cortex to most but not all thalamic nuclei. 
- Pre-ictal increases in coupling between the deep layers of the somatosensory cortex might indicate a preparation state of the epileptic focus.

- The Po is constantly driven by other thalamic nuclei during the entire SWD.

- The termination of SWD is a gradual process, which is already initiated up to 1.5 seconds prior to SWD offset.

- SWD termination involves both cortico-thalamic as well as intrathalamic processes.

- The intra-RTN communication is involved in the regulation of SWD duration.

- The rostral RTN might have a resonator function, which is crucial for SWD maintenance, whereas the caudal RTN might function as a break of this oscillatory activity (see also (Meeren et al., 2009));

- A strong directional drive from cortical focus to Po following the offset of SWD might indicate an SWD re-initiation attempt. This attempt might fail since, in contrast to SWD initiation, no reverberation circuit between cortex and Po is present.

\section{Grants}

The study was partially financed by the Donders Centre for Cognition and the BrainGain Smart Mix Program of the Netherlands Ministry of Economic Affairs and the Netherlands Ministry of Education, Culture and Science.

\section{Disclosure}

None of the authors has any conflict of interest to disclose. All authors have approved the final article.

\section{Acknowledgment}

The authors gratefully acknowledge the support of Gerard van Oijen (technical assistance); Norbert Hermesdorf (construction of electrode system units); Jos Dederen (histological verification); Hans Krijnen and Saskia Menting-Hermeling (biotechnical assistance and animal care).

\section{References}

Aker, R.G., Ozyurt, H.B., Yananli, H.R., Cakmak, Y.O., Ozkaynakci, A.E, Sehirli, U., Saka, F. Cavdar, S., Onat, F.Y., 2006. GABA(A) receptor mediated transmission in the thalamic reticular nucleus of rats with genetic absence epilepsy shows regional differences: functional implications. Brain Res. 1111, 213-221.

Berdiev, R.K., van Luijtelaar, G., 2009. Cholinergic stimulation of the nucleus basalis of Meynert and reticular thalamic nucleus affects spike-and-wave discharges in WAG/ Rij rats. Neurosci. Lett. 463, 249-253.

Bosnyakova, D. Gabova, A. Kuznetsova, G, Obukhov, Y. Midzyanovskaya, I, Salonin, D. van Rijn, C., Coenen, A., Tuomisto, L., van Luijtelaar, G., 2006. Time-frequency analysis of spike-wave discharges using a modified wavelet transform. J. Neurosci. Methods $154,80-88$.

Bosnyakova, D., Gabova, A., Zharikova, A., Gnezditski, V., Kuznetsova, G., van Luijtelaar, G. 2007. Some peculiarities of time-frequency dynamics of spike-wave discharges in humans and rats. Clin. Neurophysiol. 118, 1736-1743.

Bouwman, B.M., Suffczynski, P., Lopes da Silva, F.H., Maris, E., van Rijn, C.M., 2007. GABAergic mechanisms in absence epilepsy: a computational model of absence epilepsy simulating spike and wave discharges after vigabatrin in WAG/Rij rats. Eur. J. Neurosci. 25, 2783-2790.

Bressler, S.L., Seth, A.K., 2011. Wiener-Granger causality: a well established methodology. Neuroimage 58, 323-329.

Coenen, A.M., van Luijtelaar, E.L., 1989. Effects of diazepam and two beta-carbolines on epileptic activity and on EEG and behavior in rats with absence seizures. Pharmacol. Biochem. Behav. 32, 27-35.

Coenen, A.M., Van Luijtelaar, E.L., 2003. Genetic animal models for absence epilepsy: a review of the WAG/Rij strain of rats. Behav. Genet. 33, 635-655.

Cornwall, J., Cooper, J.D., Phillipson, O.T., 1990. Projections to the rostral reticular thalamic nucleus in the rat. Exp. Brain Res. 80, 157-171.

David, O., Guillemain, I., Saillet, S., Reyt, S., Deransart, C., Segebarth, C., Depaulis, A., 2008 Identifying neural drivers with functional MRI: an electrophysiological validation. PLoS Biol. 6, 2683-2697.
Depaulis, A., van Luijtelaar, G., 2006. Genetic modelbes of absence epilepsy. In: Pitkanen, A., Schwartzkroin, P.A., Moshe, S.L. (Eds.), Models of Seizures and Epilepsy. Elsevier Academic Press, San Diego.

Destexhe, A., 1999. Can GABAA conductances explain the fast oscillation frequency of absence seizures in rodents? Eur. J. Neurosci. 11, 2175-2181.

Dhamala, M., Rangarajan, G., Ding, M., 2008a. Analyzing information flow in brain networks with nonparametric Granger causality. Neuroimage 41, 354-362.

Dhamala, M., Rangarajan, G., Ding, M., 2008b. Estimating Granger causality from fourier and wavelet transforms of time series data. Phys. Rev. Lett. 100, 018701.

Drinkenburg, W.H., Coenen, A.M., Vossen, J.M., Van Luijtelaar, E.L., 1991. Spike-wave discharges and sleep-wake states in rats with absence epilepsy. Epilepsy Res. 9, 218-224.

Gorji, A., Mittag, C., Shahabi, P., Seidenbecher, T., Pape, H.C., 2011. Seizure-related activity of intralaminar thalamic neurons in a genetic model of absence epilepsy. Neurobiol. Dis. 43, 266-274.

Halonen, T., Pitkanen, A., Koivisto, E., Partanen, J., Riekkinen, P.J., 1992. Effect of vigabatrin on the electroencephalogram in rats. Epilepsia 33, 122-127.

Huguenard, J.R., McCormick, D.A., 2007. Thalamic synchrony and dynamic regulation of global forebrain oscillations. Trends Neurosci. 30, 350-356.

Kaufman, E.F., Rosenquist, A.C., 1985. Afferent connections of the thalamic intralaminar nuclei in the cat. Brain Res. 335, 281-296.

Kostopoulos, G.K., 2001. Involvement of the thalamocortical system in epileptic loss of consciousness. Epilepsia 42 (Suppl. 3), 13-19.

Lally, N., Mullins, P.G., Roberts, M.V., Price, D., Gruber, T., Haenschel, C., 2014. Glutamatergic correlates of gamma-band oscillatory activity during cognition: a concurrent ERMRS and EEG study. Neuroimage 85 (Pt. 2), 823-833.

Liu, X.B., Jones, E.G., 1999. Predominance of corticothalamic synaptic inputs to thalamic reticular nucleus neurons in the rat. J. Comp. Neurol. 414, 67-79.

Liu, X.B., Coble, J., van Luijtelaar, G., Jones, E.G., 2007. Reticular nucleus-specific changes in alpha3 subunit protein at GABA synapses in genetically epilepsy-prone rats. Proc. Natl. Acad. Sci. U. S. A. 104, 12512-12517.

Lüttjohann, A., van Luijtelaar, G., 2012. The dynamics of cortico-thalamo-cortical interactions at the transition from pre-ictal to ictal LFPs in absence epilepsy. Neurobiol. Dis. 47, 49-60.

Lüttjohann, A., Zhang, S., de Peijper, R., van Luijtelaar, G., 2011. Electrical stimulation of the epileptic focus in absence epileptic WAG/Rij rats: assessment of local and network excitability. Neuroscience 188, 125-134.

Lüttjohann, A., Schoffelen, J.M., van Luijtelaar, G., 2013. Peri-ictal network dynamics of spikewave discharges: phase and spectral characteristics. Exp. Neurol. 239, 235-247.

Marescaux, C., Vergnes, M., Depaulis, A., 1992. Genetic absence epilepsy in rats from Strasbourg-a review. J. Neural Transm. Suppl. 35, 37-69.

Maris, E., Oostenveld, R., 2007. Nonparametric statistical testing of EEG- and MEG-data. J. Neurosci. Methods 164, 177-190.

Maris, E., Bouwman, B.M., Suffczynski, P., van Rijn, C.M., 2006. Starting and stopping mechanisms of absence epileptic seizures are revealed by hazard functions. J. Neurosci. Methods 152, 107-115

Maris, E., Schoffelen, J.M., Fries, P., 2007. Nonparametric statistical testing of coherence differences. J. Neurosci. Methods 163, 161-175.

Meeren, H.K., Pijn, J.P., Van Luijtelaar, E.L., Coenen, A.M., Lopes da Silva, F.H., 2002. Cortical focus drives widespread corticothalamic networks during spontaneous absence seizures in rats. J. Neurosci. 22, 1480-1495.

Meeren, H., van Luijtelaar, G., Lopes da Silva, F., Coenen, A., 2005. Evolving concepts on the pathophysiology of absence seizures: the cortical focus theory. Arch. Neurol. 62, 371-376.

Meeren, H.K., Veening, J.G., Moderscheim, T.A., Coenen, A.M., van Luijtelaar, G., 2009. Thalamic lesions in a genetic rat model of absence epilepsy: dissociation between spike-wave discharges and sleep spindles. Exp. Neurol. 217, 25-37.

Oostenveld, R., Fries, P., Maris, E., Schoffelen, J.M., 2011. FieldTrip: open source software for advanced analysis of MEG, EEG, and invasive electrophysiological data. Comput. Intell. Neurosci. 2011, 156869.

Paxinos, G., Watson, C., 1998. The rat brain in stereotaxic coordinates. Academic Press, SanDiego.

Percival, D.B., Walden, A.T., 1993. Spectral analysis for physical applications: multitaper and conventional univariate techniques. Cambridge University Press, Cambridge.

Pinault, D., O'Brien, T.J., 2005. Cellular and network mechanisms of geneticallydetermined absence seizures. Thalamus Relat. Syst. 3, 181-203.

Polack, P.O., Guillemain, I., Hu, E., Deransart, C., Depaulis, A., Charpier, S., 2007. Deep layer somatosensory cortical neurons initiate spike-and-wave discharges in a genetic model of absence seizures. J. Neurosci. 27, 6590-6599.

Proulx, E., Leshchenko, Y., Kokarovtseva, L., Khokhotva, V., El-Beheiry, M., Snead III, O.C., Perez Velazquez, J.L., 2006. Functional contribution of specific brain areas to absence seizures: role of thalamic gap-junctional coupling. Eur. J. Neurosci. 23, 489-496.

Seidenbecher, T., Pape, H.C., 2001. Contribution of intralaminar thalamic nuclei to spikeand-wave-discharges during spontaneous seizures in a genetic rat model of absence epilepsy. Eur. J. Neurosci. 13, 1537-1546.

Sitnikova, E., Dikanev, T., Smirnov, D., Bezruchko, B., van Luijtelaar, G., 2008. Granger causality: cortico-thalamic interdependencies during absence seizures in WAG/Rij rats. J. Neurosci. Methods 170, 245-254.

Sohal, V.S., Keist, R., Rudolph, U., Huguenard, J.R., 2003. Dynamic GABA(A) receptor subtype-specific modulation of the synchrony and duration of thalamic oscillations. J. Neurosci. 23, 3649-3657.

Steriade, M., 1998. Corticothalamic networks, oscillations, and plasticity. Adv. Neurol. 77, $105-134$

Steriade, M., 2003. Neuronal substrates of sleep and epilepsy. Cambridge University Press, Cambridge.

van Luijtelaar, G., Sitnikova, E. Lüttjohann, A. 2011. On the origin and suddenness of absences in genetic absence models. Clin. EEG Neurosci. 42, 83-97. 